\title{
Significance of single-cell and spatial transcriptomes in cell biology and toxicology
}

\author{
Duojiao Wu • Xiaozhuan Liu • Jiaqiang Zhang $\cdot$ Li Li • \\ Xiangdong Wang
}

Received: 12 September 2020 / Accepted: 1 December 2020 / Published online: 4 January 2021

(C) The Author(s), under exclusive licence to Springer Nature B.V. part of Springer Nature 2021

Single-cell RNA sequencing (scRNA-seq) is a new approach to demonstrate transcriptional profiles, cell-cell communication, and intracellular regulation in an isolated cell, which loses the location information in the tissue. Single-cell and spatial transcriptomics address the tissue location where transcriptomic profiles and interactions of special cell subpopulations occur, the intercellular neighborhoods and how cell morphological and molecular phenomes are functionally correlated, and the setting where newly discovered lineages and populations biologically interact through combining scRNA-seq with reconstructed spatialization. scRNA-seq has a great

Duojiao Wu and Xiaozhuan Liu contributed equally to this work.

D. Wu $\cdot$ X. Wang $(\bowtie)$

Zhongshan Hospital Institute for Clinical Science, Shanghai Institute of Clinical Bioinformatics, Shanghai Engineering Research for AI Technology for Cardiopulmonary Diseases, Zhongshan Hospital, Fudan University, Shanghai, China; Jinshan Hospital Centre for Tumor Diagnosis and Therapy, Jinshan Hospital, Fudan University, Shanghai, China e-mail: xdwang@fuccb.com

\section{Liu $\cdot$ J. Zhang $\cdot$ L. Li $(\bowtie)$}

Clinical Center for Single Cell Biomedicine, People's Hospital of Zhengzhou University, Zhengzhou, China

e-mail: hnykylili@126.com

\section{J. Zhang $(\bowtie)$}

Department of Anesthesiology and Perioperative Medicine, Henan Province People's Hospital, People's Hospital of Henan University, People's Hospital of Zhengzhou University,

Zhengzhou, China

e-mail: jqzhang@henu.edu.cn impact on understanding transcriptional networks and interactions in single-cell responses to drugs, e.g., sensitivity and toxicity (Wang et al. 2018; Song et al. 2019). Single-cell and spatial transcriptomics may provide greater insights on networks and function of transcriptional regulations of special cell populations within tissue architecture when cells interact with drugs, although the understanding of spatial transcriptomics in toxicology remains limited. Spatial transcriptomics experienced from manipulated positional information (e.g., cryosection, laser capture microdissection, imaging), in situ positional RNA expression (e.g., osmFUSH, multiplexed error-robust fluorescence in situ hybridization, barcodebased spatialization), and spatial reconstruction from scRNA-seq (Teves and Won 2020). scRNA-seq-based spatial transcriptomics is considered as a critical part of single-cell biomedicine to provide positional information on functional communication and interaction between cells and to reconstruct the spatial architecture of target cell-oriented neighbors and microenvironments (Wang and Wang 2020; Zeng et al. 2020). Of those, molecular mechanisms of single-cell spatial responses to drugs become a new and important topic in the Cell Biology and Toxicology and will provide deeper insights in understanding positional intercellular communication during alterations of cell sensitivity, tolerance, and resistance against drugs and therapies.

Values of single-cell and spatial transcriptomics are highly dependent upon the designs and strategies of studies. For example, spatial transcriptomic profiles of target cells can be monitored during various stages of cell colonization, migration, differentiation, phenome transit, 
and response to drugs. McFaline-Figueroa et al. (2019) made a systemic design to study the intracellular network signals of each single-cell and intercellular communications between single cells during epithelial-tomesenchymal transition by profiling single-cell transcriptomes. This is an example of monitoring transcriptional phenotypes, interactions, and regulations dynamically and spatially in the processes of cell differentiation from progenitors to mature cells and cell transition from epithelial/endothelial states to mesenchymal phenomes. Regulatory roles of target genes and molecules can be further defined in the process of cell-to-cell transition through integration with target gene editing. The reliability of single-cell-based spatial transcriptomes depends upon accurate methodologies of pseudospatial trajectory analyses and alignments and upon dynamic specificities of biological models and responses. Baccin et al. (2020) presented the location and organization of Cxcl12-abundant-reticular cell subsets (Adipo-CAR and Osteo-CAR) on sinusoidal and arteriolar surfaces as "cytokine-secreting cells" responsible for the construction of perivascular micro-niches, by combining scRNA-seq with spatial transcriptomes. Single-cell transcriptomes are more focused on transcriptional profiles of target cell populations at a single-cell level. Pseudospatial trajectory locations request transcriptional profiles of all cell populations at the single-cell level, including formed all clusters corresponding to distinct cell types or stages of differentiation. Target single cells can be selected and isolated by manual means, multiplexing, flow cytometer, integrated fluidic circuits, liquid handling robotics, nanociroplets, picowells, or in situ barcoding (Fig. 1a). The success rate of single-cell isolation and purification is highly dependent upon the nature of organs/tissues, the accuracy of target cell area, the procedure of tissue harvest, and the quality of sample transport and maintenance in patients. RNA library and sequencing of target single cells can be performed after the quality of purified and amplified RNA is confirmed, to measure expression profiles and transcriptomes, define cell type categories, and identify new cell types (Fig. 1b). The transcriptional profiles of isolated single cells lack the information of tissue construction, but tissue visualization and neighbor structure can be reconstructed by mapping expressions of cellular surface receptor/ligand genes expressions, pseudo-imaged by pseudotime analysis and clustering/ denoising, or automatically analyzed using computational mode after latent manifold learning (Fig. 1c). The analytic mode of artificial intelligence is based on dimension data, point distance, distance affinity, local relationship, and underlying manifold. The local and global nonlinear structure can be configurated using the information-geometric distance between data points and furthermore visualized with the potential of heatdiffusion for affinity-based transition embedding (PHATE) and t-distributed stochastic neighbor embedding (t-SNE) (Fig. 1d). Pseudospatial trajectory locations and pseudospatial transcriptomes of cell populations, especially rare cell populations, can be visualized using scRNA-seq data based on one-dimensional t-SNE for simultaneously visualizing the expression patterns of thousands of genes (Fig. 1e).

Spatial transcriptomes demonstrate transcriptional profiles with the definite location in tissues by loading tissue sections on slides with barcoded poly(d)T oligonucleotides. Vickovic et al. (2019) developed a method to measure the high-definition spatial transcriptomics and capture RNA from histological tissue sections with several hundred thousand transcript-coupled spatial barcodes at $2-\mu \mathrm{m}$ resolution. The first step of spatial transcriptomics is to select target areas and locations and prepare multiple tissue cryosections (Fig. 1f), which decide the quality of biological and pathological cell organization. Optimal multi-sections of organ/tissue can be dynamically selected in an in vivo model, although the quality of selected sections in the human is hard to maintain due to the variation of clinical phenomes, the priority of pathology, and the experience of the clinician. The frozen tissue section was prepared for in situ sequencing, and hematoxylin-eosin-stained sections for morphology, morphometric analysis, and calculated coordinates to identify the location sport of RNA expression. The quality of spatial transcriptomics is highly dependent upon the size of array wells, load of beads, and quality of oligos on the bead surface, of which each contains poly(d)TVN, UMI, spatial barcode, adaptor, promoter, and cleavage site (Vickovic et al. 2019). Oligo-dT probes and template-switching oligos are designed based on different sequencing methods (e.g., SMART-seq, SMART-seq2, SCRB-seq, msSCRB-seq, CEL-seq, Drop-seq, Microwell-seq, or Split-seq). For example, SMART-seq has the same sequence at the $3 / 5$ end which needs one probe for amplification of double-chain cDNA and the $\mathrm{rGrG}+\mathrm{G}$ at the end of template-switching oligos to accelerate the stability and specificity, while SCRB-seq, in addition, has UMI and Barcode on oligo-dT probes similar to what the CEL-seq2 has. 


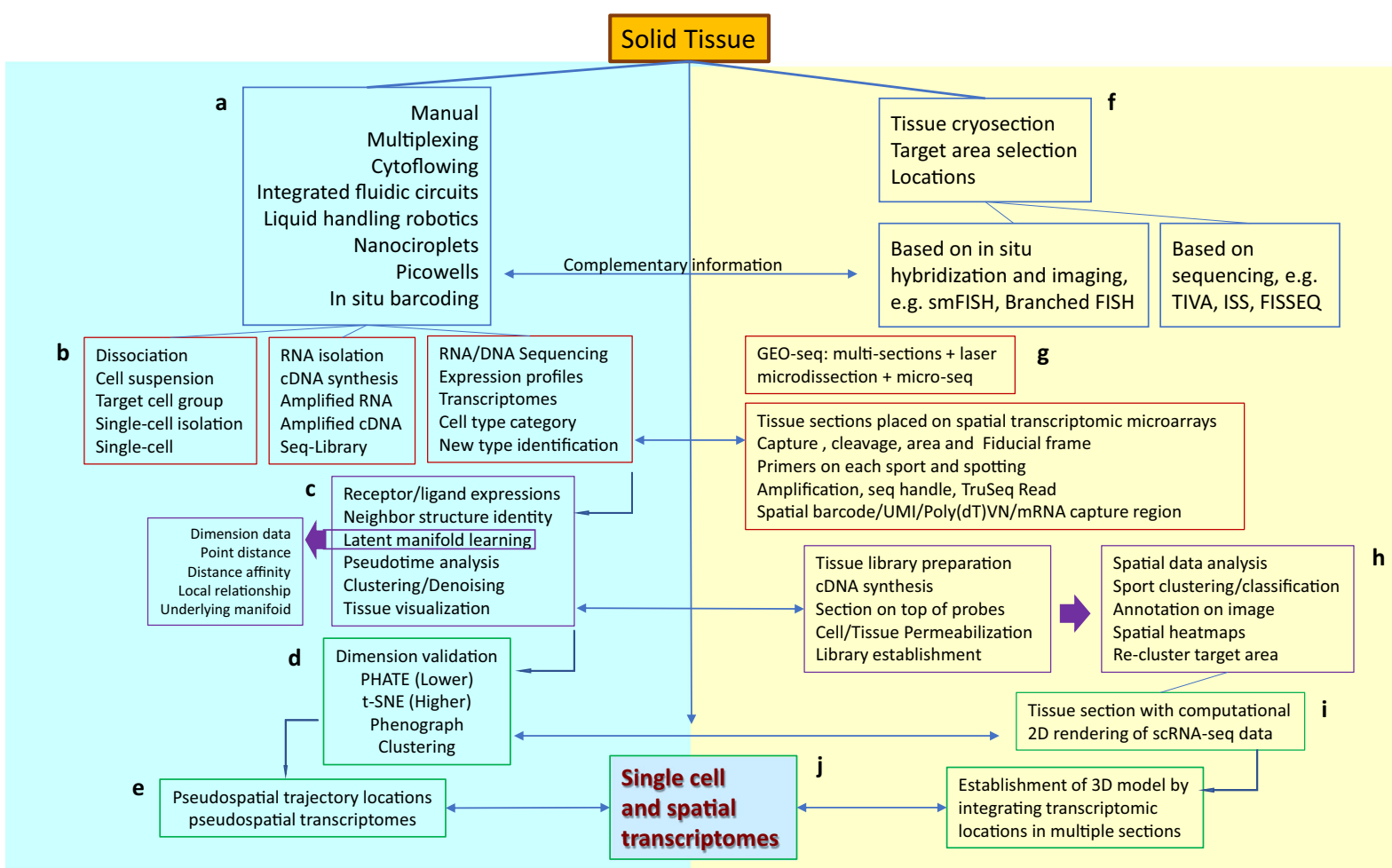

Fig. 1 Principle analyses of single-cell and spatial transcriptomics. a Selection and isolation of target single cells. b RNA library and sequencing of target single cells, c Reconstruction of tissue visualization and neighbor structure by expressions of cellular surface receptor/ligand genes expressions, pseudotime analysis and clustering/denoising, and computational mode. d Configuration of local and global nonlinear structures using the informationgeometric distance between data points and furthermore visualized with the potential of heat-diffusion for affinity-based transition embedding (PHATE) and t-distributed stochastic neighbor embedding (t-SNE). e Pseudospatial trajectory locations and pseudospatial transcriptomes of cell populations. f Preparation of multiple tissue cryosections. g Combination laser capture microdissection with scRNA-seq to define spatial transcriptomic

With the rapid development of sequencing methods, the accuracy of in vivo and in situ position reconstructed from scRNA-seq has been improved significantly, including spatial transcriptomic microarrays, capture, cleavage, area, fiducial frame, primers on each sport and spotting, amplification, seq handle, TruSeq read, and spatial barcode/ $\mathrm{UMI} / \operatorname{Poly}(\mathrm{dT}) \mathrm{VN} / \mathrm{mRNA}$ capture region (Fig. 1g). GEO-seq is a method of combining laser capture microdissection with scRNA-seq in multi-sections and defining spatial transcriptomic profiles in a small number of target cells (Chen et al. 2017; Peng et al. 2020). GEO-seq focuses more on pathology-specific spatial transcriptomes to explore cell differentiation, evolution, fate, biology, and gene regulatory networks. The high-definition spatial profiles. h Annotation on image, spatial heatmaps, and reclustered target area. i Establishment of 2D and 3D models. j The full picture of single-cell and spatial transcriptomics by integrating gene expression and tissue image. Abbreviations: Dropseq droplet sequencing, FACS fluorescence-activated cell sorting, Geo-seq geographical position sequencing, PAGA partition-based graph abstraction, PCA principal component analysis, SCENIC single-cell regulatory network inference and clustering, scRNAseq single-cell RNA sequencing, seqFISH sequential fluorescence in situ hybridization, Slide-seq slide sequencing, SPLiT-seq split pool ligation-based transcriptome sequencing, UMAP uniform manifold approximation and projection, and WGCNA weighted gene coexpression network analysis

transcriptomics was designed to identify gaps in spatial position and great biases in cell recovery from scRNA-seq and needs of pre-selected markers and standardization, in order to improve spatial resolution, scalability, or applicability. During those processes, the tissue library preparation and establishment are dependent upon probe designs, cDNA synthesis, specificity, and release of RNA from permeabilized cells/tissues. Compared to scRNA-seq, tissue spatial transcriptomes has relatively limited spatial data and sports numbers for spatial analysis, clustering/classification, while providing more exact annotation on the image, spatial heatmaps, and re-cluster target area (Fig. 1h). With the aid of computational data-analysis strategy, 2D and $3 \mathrm{D}$ models can be established by 
integrating transcriptomic locations in multiple sections (Fig. 1i). The full picture of single-cell and spatial transcriptomics will be visualized by integrating gene expression of isolated target cells from scRNA-seq with gene expression and image of tissue cells from LCM, LCM-seq data, enriching population marker genes in locations, and reconstructing single cell-based spatial transcriptomic profiles and morphologies (Fig. 1j). scRNA-seq alone defines the biological characterization of target cell types, while single-cell and spatial transcriptomics identify spatial locations of target cell types previously known/unknown. It is also important to evaluate the specificity and accuracy of corresponding structure, localization, and connection of cells by tracing labeled molecules, matching gene expression of receptors and ligands, and counting the frequency of cell populations from scRNA-seq and LCM-seq, respectively. Spatial locations, relationships, and biological functions of known/unknown cell types should be furthermore confirmed with specific biomarkers of the individual populations, e.g., production of mediators/factors, intercellular communications, or neighborhood relations.

Single-cell and spatial transcriptomics are a new alternative to deeply understand molecular mechanisms of the disease dynamically and multi-dimensionally, develop new classes of spatial biomarker panels to diagnose diseases and monitor the progression and therapeutic effects, and discover new categories of spatial targetbased drugs. Single-cell and spatial transcriptomics have been applied to understanding spatial organizations and locations of the tumor microenvironment, cancer cell progression, reconstructed morphology, and intercellular interactions. With rapid development and improvement, singe-cell and spatial transcriptomics can provide a new approach in molecular pathology to identify spatial gene expression and transcriptional profiles; uncover cell-cell interaction, communication, and signaling in tissue; and provide a new form of pathological diagnosis. Baccin et al. (2020) demonstrated transcriptional profiles of bone-marrow-resident cell types, defined those cell localizations and spatial sources of prohematopoietic factors, and reconstructed the threedimensional bone-marrow organization by single-cell and spatial transcriptomics. Bone-marrow single-cell and transcriptomics can be used to dynamically understand spatial locations of bone-marrow-resistant cell types and spatial sources of inflammatory mediators and growth factors as well as to multi-dimensionally monitor alterations of cell neighborhoods and connections in the development of disease and in response to therapy. Aoki et al. (2020) firstly demonstrated spatial phenomes and characteristics of immune cells and mediators within the Hodgkin lymphoma microenvironment and spatial interactions among those immune cells by scRNA-seq and multiplexed spatial assessment. This is an example of discovering diagnostic biomarkers and therapeutic targets for immunotherapy through spatial functions and characters of target cell populations and intercellular communication.

It is a new opportunity and mission for us to introduce and translate single-cell and spatial transcriptomics into clinical research and trials, although there are many challenges to be overcome. The methodology of scRNA-seq is on its way to be translated into clinical application to identify rare cells, previously unknown cell types and subtypes in normal and diseased tissue. The major challenges of scRNA-seq in clinical application are the selection of cell populations, standardization of sample handles, analysis of bioinformatics, and high cost of measurements. The combination of scRNA-seq and spatial transcriptomics has more challenges and difficulties to be faced, e.g., the complexity of sampling and handling, compatibility of multi-tissues, definition of target tissue areas, stability of technology and performance, and cost overload per sample. It is also a challenge to furthermore understand the scientific values and significance of tissue functional organization and cell-to-cell interactions in the microenvironment at the single-cell level. Although it is still far away from direct use in clinical diagnosis and therapy, single-cell and spatial transcriptomics is becoming more important in cell biology, biomedicine, and system biomedicine.

In conclusion, single-cell and spatial transcriptomics are a new approach to explore spatial transcriptomic profiles and cell-cell interactions in tissues, spatial relations between cell locations and transcriptional phenomes, and evolutions of lineages and populations. Scientific values of single-cell and spatial transcriptomics can be utilized for study strategies, clinical questions, and potential applications. In addition to a deeper understanding of multi-dimensional mechanisms of cell function, single-cell and spatial transcriptomics will provide insights into spatial responses of target cells to precision medications.

\section{Compliance with ethical standards}

Conflict of interest The authors declare that they have no conflict of interest. 
Ethical approval Not applicable.

Consent to participate Not applicable.

Consent for publication Not applicable.

Availability of data and materials Not applicable.

Code availability Not applicable.

\section{References}

Aoki T, Chong LC, Takata K, Milne K, Hav M, Colombo A, et al. Single-cell transcriptome analysis reveals disease-defining Tcell subsets in the tumor microenvironment of classic Hodgkin lymphoma. Cancer Discov. 2020;10(3):406-21. https://doi.org/10.1158/2159-8290.CD-19-0680.

Baccin C, Al-Sabah J, Velten L, Helbling PM, Grünschläger F, Hernández-Malmierca $\mathrm{P}$, et al. Combined single-cell and spatial transcriptomics reveal the molecular, cellular and spatial bone marrow niche organization. Nat Cell Biol. 2020;22(1):38-48. https://doi.org/10.1038/s41556-0190439-6.

Chen J, Suo S, Tam PP, Han JJ, Peng G, Jing N. Spatial transcriptomic analysis of cryosectioned tissue samples with Geo-seq. Nat Protoc. 2017;12(3):566-80. https://doi. org/10.1038/nprot.2017.003.

McFaline-Figueroa JL, Hill AJ, Qiu X, Jackson D, Shendure J, Trapnell C. A pooled single-cell genetic screen identifies regulatory checkpoints in the continuum of the epithelial-tomesenchymal transition. Nat Genet. 2019;51(9):1389-98. https://doi.org/10.1038/s41588-019-0489-5.

Peng G, Cui G, Ke J, Jing N. Using single-cell and spatial transcriptomes to understand stem cell lineage specification during early embryo development. Annu Rev Genomics Hum Genet. 2020;21:163-81. https://doi.org/10.1146 /annurev-genom-120219-083220.

Song D, Yang D, Powell CA, Wang X. Cell-cell communication: old mystery and new opportunity. Cell Biol Toxicol. 2019;35(2):89-93. https://doi.org/10.1007/s10565-01909470-y.

Teves JM, Won KJ. Mapping cellular coordinates through advances in spatial transcriptomics technology. Mol Cells. 2020. https://doi.org/10.14348/molcells.2020.0020.

Vickovic S, Eraslan G, Salmén F, Klughammer J, Stenbeck L, Schapiro D, et al. High-definition spatial transcriptomics for in situ tissue profiling. Nat Methods. 2019;16(10):987-90. https://doi.org/10.1038/s41592-019-0548-y.

Wang W, Gao D, Wang X. Can single-cell RNA sequencing crack the mystery of cells? Cell Biol Toxicol. 2018;34(1):1-6. https://doi.org/10.1007/s10565-017-9404-y.

Wang W, Wang X. A refocus on the advances of single cell biomedicine. Cell Biol Toxicol 2020.

Zeng Y, Wang X, Zhang J. Single-cell biomedicine: roles of single-cell nuclear elements. Cell Biol Toxicol. 2020;36(1): 1-3. https://doi.org/10.1007/s10565-020-09515-7.

Publisher's note Springer Nature remains neutral with regard to jurisdictional claims in published maps and institutional affiliations. 